\title{
The Effectiveness of Static and Dynamic Stretching on Hamstring Flexibility after 4-Weeks Training to Prevent the Risk of Injuries
}

\author{
Sismaherni bt Suardi Shaharuddin ${ }^{1}$, Srinivas Mondam ${ }^{2 *}$
}

${ }^{1}$ Sports Rehabilitation student, Faculty of Sports Sciences and Coaching, Sultan Idris University of Education, Tanju Malim, Perak 35900, MALAYSIA

${ }^{2}$ Lecturer, Sports Rehabilitation, Faculty of Sports Sciences and Coaching, Sultan Idris University of Education, Tanju Malim, Perak 35900, MALAYSIA

*Email: vasmpt@gmail.com

\begin{abstract}
This study was conducted to investigate the effect of static and dynamic stretching on hamstring muscle flexibility and risk of injury. A total of 20 female students who are in their final year of various departments at the Sultan Idris of Educational University (UPSI) were involved in this study. Subjects were selected based on the body mass index and never active in sports. The will divided into two groups. Group A $(n=10)$ will perform five types of static stretching exercise while the second group B $(n=10)$ will perform five types of dynamic stretching exercise. They will perform 5-minutes warming up exercise that is jogging before start the stretching. The stretching exercise will handle in three days alternately fo four weeks. The flexibility of the hamstring muscles was measured using a test range protrudes. The instrument that used to measure the flexibility of the hamstring muscles sit reach box, and it is measure before and after the intervention. Pre-test shows no differences between both types of stretching exercise. For the post-test score, static stretching exercise shows the better hamstring flexibility scores than dynamic stretching exercise, $t(9)=23.60, p<0.005$. The results indicated that static stretching exercise is more effective in increasing the hamstring flexibility compared to dynamic stretching exercise and also have results on it in reducing the risk of injury.
\end{abstract}

Keywords: Flexibility, un-sport female student, static stretching, dynamic stretching, risk of injury

\section{INTRODUCTION}

The flexibility is the one of fitness component. Who has well in flexibility can bend their joints at the full range of motion without pain. Some of the opinion was define it as the joint flexibility may be the factor to an injury to be occurring. If less flexibility in the joint, then this will cause less range of motion. However, what is meant by flexibility? Flexibility is the ability to extend the muscles to allow a joint or many joints to move based on the full range of motion. This statement is supported by Zachezeweskil (1) which is he has defined muscle flexibility as the muscle's ability to lengthen to allow a joint or more than one joint to move in full range of motion. The ability of a muscle to return back to normal length is the meaning of loss of muscle flexibility. This condition will make the range of motion of the joint reduce (1). We need the flexibility to improve the range of motion, the muscle and other connective tissues around the joint should be stretched. Both flexibility and range of motion is interrelated. When 
we stretch the connective tissues surrounding the joints, the range of motion will increase. Here, we can see, by stretching, the range of motion can improve.

Based on other studies, the flexibility is the range of motion and it is play a role as a general health-related of physical fitness components and daily activities is included as well as sports activities (2). In the other side, flexibility is influenced by several factors. Among these are gender, age, the stiffness of joints, joints and tendons. According to Mohammad Thiet. al., 2011, gender, age, immoderate adipose tissue, skin, stiff muscle, ligaments, and tendons are factors impacting muscle flexibility and joint ROM. People do not realize that the flexibility was having different of types. Type of flexibility can be grouped according to the category of sport performed. Among the types of flexibility that exists is the dynamic flexibility that is conducted with movement and static flexibility done without movement. According to Kruz, flexibility can be divided into three, which are dynamic flexibility, static active flexibility, and static passive flexibility. Dynamic flexibility means the ability to perform dynamic movements of muscles to bring a limb through its full range of motion in joints. This kind of flexibility is known as kinetic flexibility. Static-active flexibility also called as active flexibility. Static-active flexibility means the ability to receive and maintain the extended position using only tension agonist and antagonist synergist during stretched. For the example is lifting a leg up without external assistance (other than your own feet). Passive static flexibility is recognized as passive flexibility. This kind of flexibility means assume the position is stretching and then maintain them using the weight, support your limbs or by using other tools. Notice that ability to keep the position does not come solely from your muscles, as it does with static-active flexibility. For example is doing splits. Researchers have shown that active flexibility is closely related to the level of sports achievement than passive flexibility. Active flexibility is difficult to set up compared to static flexibility. To take over the position of the initial extension, the active flexibility need passive flexibility and strength of the muscle is to maintain and hold the position.

Therefore, to avoid an athlete injured due to the flexibility of muscles, several steps must be taken which is by doing stretching exercises. Stretching exercises also have different types. Among these are static stretching exercises, ballistic exercises, proprioceptive neuromuscular facilitation (PNF) and dynamic stretching exercises. Flexibility is also often associated with all kinds of sports activities. This is supported by Mohammad Thiet. al,. 2011, where flexibility can be achieved and maintain from static, dynamic, ballistic and PNF stretching exercise. Also, this type of workout can also be used to reduce injury, improve performance, increase power and strength. However, in this study, I only emphasize only two types of exercise to improve and build muscle flexibility. So, what meant by static stretching? According to M. Alter, static stretching is the stretching of the muscle or the group of muscle to reaching the maximum points and then maintaining and holding at that position (3).

Static stretching exercise is a kind of exercise that is done in a static state without any additional movement other than the motion of the muscle stretch. Stretching exercise that that are performed without movement is refer to static stretch. Besides that, static stretches cause the individual at the stretch and hold position for specific amount of time. (http://injuryfix.com/archives/stretching.php). Static stretching is also an old workout, otherwise known as traditional stretching exercises.

Based on Young and Behm, 2002 studies, static stretching is the basic part of a warm-up for decades. This type of stretching is performed before they start with specific sports activities. Most people choose these exercises because this exercises safer and also less potential for injury. C. D. Weiner et.al., 2003 state that stretching is one of the safest and most commonly performed stretching methods used to measure muscle length. This exercise is easy to do and does not require additional equipment during implementation. Same as Stephen B. J, Gilchrist, Donna F. and C. Dexter, 2004 state the static stretching is the easiest and most frequently used as stretching method, each approach has advocates. Most of the athletes are advised to do this kind of stretching exercises before performing certain sports activities. Also other than reducing injury, static stretching also can reduce muscle soreness, and also improve performance. Based on studies of Safran et.al., 1989, Smith 1994, High et.al., 1989, Young and Behm, 2002 and Young 2007, they state that, static stretching is benefit in reducing and preventing the injury, reduce the muscle soreness and enhance the performance. But, in several studies, they concluded that static stretching does not give any results in injury prevention (4). Although some of research finds that the most flexible person has high potential to getting injuries than the moderately flexible individual (4). Furthermore, the early decade research shows that static stretching can improved the performance $(4,7)$. Based on recent research found that static stretching will decrease the different strength and power output test (7). Static stretching exercise is often used for increase the muscle length. Usually, strength and conditioning specialist and also athlete will use static stretching as a common stretching to improve the muscle strength (D. Scott, Paul E., Kristi L. Mccale, Jerry A., and Jaime M., 2005). The second stretching exercises that involved in this study are dynamic stretching exercises. Dynamic stretching is define as a stretch to lengthen the muscle and performed by the movement. According to Kurz, dynamic stretching is involves moving parts of your body and gradually increasing reach, speeds of movement, or both (9). Compared to static stretching, it is only focus on one movement only. In contrast of static stretching, dynamic stretching 
involves the whole body motion and incorporates actively and rhythmically contracting muscle through part of it is functional range of motion (10). Based on flexibility, dynamic stretching exercise also can help to increase the flexibility of muscle. Besides this type of stretching also can be used as an exercise to prepare the muscle before start the specific sports activities. But, the disadvantages of this stretching, it may be can increase the risk of injury because the present of addition movement during perform the stretching exercise. Even though, some of research found, the dynamic stretching exercise is more effective compared than the static exercise in improving muscle flexibility.

In the studies of Fredrick G. A., 2000, they found the dynamic stretching can stimulates the nervous system and decreasing the potential of injury, rising the core temperature, muscle temperature and lengthen the muscles. In the other studies by Faigenbaum et. al., 2005, they compared between dynamic warm-up and static stretching in different age group and variety athlete and the results shows the dynamic warm-up will rising the flexibility and enhanced the performances among children for vertical jump. At the same time, dynamic warm-up also can improve long-jump performance. Based on my study, I try to investigate which exercise is better to increasing flexibility and reducing injuries. From the previous study, some of researcher said, static stretching is the best exercise for increase the flexibility of the muscle. The same results found in the studies of David G.Behm and Anis Chaouachi, 2011. They notice that static stretching effectual in increasing range of motion of the joint (4) and can reduce the propensity of getting the injuries (4) able to avert from injury (Smith 1994), can reduce muscle soreness (4) and enhanced the performance (4).

But, some of researcher said the opposite. They said the static stretching exercise give the negative effect on performance (2). And at the same time, they suggest static stretching exercise is better performed as cool down exercise rather than be a warm-up exercise because static exercise may cause muscle cool down (11). Compared to dynamic stretching exercise, most of researcher said, dynamic stretching exercise can improve performance, power, and suitable be a warming up exercise compared to static stretching exercise. One of study by Fredrick G.A., 2005 has shown the effectiveness of dynamic stretching exercise in increasing muscle flexibility; increase in muscle and core temperature and at the same time can reduce the chances of injury. In other study, they found dynamic stretching exercise can improve flexibility and also improved performance of children in vertical jump (11). It is quite enough to say; both stretching exercises can improve muscle flexibility and reduce the chance of injury.

\section{Procedure}

The procedures of the experiment were explained well to every subject. A form was distributed to each subject before they involved in the exercise of this research. This form is a consent form for the candidates to become a subject in the study. The participants were divided into two groups: group exercise static stretching, dynamic stretching exercise group. For the first group, there are five types of exercises hamstring muscles stretching to perform. The stretching performed in static conditions, standing upright, does not involve any additional movement. Whereas for the second group, will perform dynamic stretching in motion. They need to move approximately $20 \mathrm{~m}$ or do ten repetitions alternately for both lower limbs go and back. The second group also performed five types of exercise for this group. Flexibility hamstring muscle taken before and after stretching exercise conducted. All data is record in the table. The study design is an experimental that involved pre-test and post-test. The study will be conducted in 4 weeks, three days alternately per week. The study includes 20 female noathlete students of UPSI. The data was recorded before and after stretching. The study randomized controlled trial to determine which stretching exercise may increase the score of hamstring flexibility after 4-weeks intervention and reduce the risk of injury. Inclusion criteria in this study are the age range of the subjects between 20-25 years old. Besides that, the subject was selected based on their body mass index scores. Only the persons with normal body mass index will be selected. The study is conducted on non-athlete population.

For the exclusion criteria, I did not choose athlete as my subject. The subject with the less or more body mass index score is not qualified to be subject for this study. Male subjects also not participate in this study. The study population was 20 female non-athlete UPSI students. They are the final year students from various courses in UPSI that has the knowledge of static and dynamic stretching exercises. The subjects are randomly selected because we have a lack of time to find the subject. This research is not only focused on the professional athlete but also involve the recreational athlete. When collecting the data, all the athletes involved are not having the health problem or injuries. The venues of the research are located at UPSI. Netball court at Zaaba College of UPSI is the places to performing the stretching. There is some instrument needed during the test that used to collect data and also exercise. Therefore, I chose sit-and-reach boxes to measure the flexibility of the hamstring muscles. Besides that, paper and pen are used to record down all the results has been taken. In the other hand, I used measuring tape to measure $20 \mathrm{~m}$ long and putting powder as marking point at the end of $20 \mathrm{~m}$ for the purpose of testing the stretching exercises. 


\section{DATA ANALYSIS}

All data collected is required to analyze to obtain the best result by using the IBM Statistical Package for Social Sciences (SPSS) version 20. The data is analyzed by using paired sample t-test and one sample $t$-test. The descriptive analysis was used to look over the weight and height of the subjects. The level of significant is $p<0.005$.

\section{RESULTS}

Table 1

\begin{tabular}{|l|l|c|c|c|c|}
\hline & & Mean N Std. & Mean N Std. & Mean N Std. & Mean N Std. \\
\hline & & Deviation Std. & Deviation Std. & Deviation Std. & Deviation Std. \\
\hline & & Error Mean & Error Mean & Error Mean & Error Mean \\
\hline Pair 1 & Pre-Test Score Dynamic Stretching & 28.3900 & 10 & 4.87908 & 1.54290 \\
\hline & Post-Test Dynamic Stretching & 31.0700 & 10 & 4.16335 & 1.31657 \\
\hline Pair 2 & Pre-Test Score Static Stretching & 30.2500 & 10 & 10.08081 & 3.18783 \\
\hline & Post-Test Score Static Stretching & 32.6500 & 10 & 10.68283 & 3.37821 \\
\hline
\end{tabular}

Table 1 above shows the results of paired sample -t test analysis for the mean score of the hamstring muscles flexibility after doing dynamic stretching exercises after 4 weeks training three times a week increased compared to the previous the ones. The score hamstring muscle flexibility before doing dynamic stretching exercises for 4 weeks is $(\mathrm{M}=28.39, \mathrm{SD}=4.88)$, while the score after doing dynamic stretching exercises for 4 weeks is $(\mathrm{M}=31.07, \mathrm{SD}=$ 4.16). Based on Table 1, the results of paired samples t-test analysis found that scores the flexibility of the hamstring muscles after exercise static stretching exercises for four weeks is increased. The mean of scores before doing stretching exercises is $(\mathrm{M}=30.25, \mathrm{SD}=8.10)$. While the men value for the score after the exercise is dynamic stretching exercises $(\mathrm{M}=32.65, \mathrm{SD}=10.68$.

Table 2

\begin{tabular}{|l|c|c|c|c|c|}
\hline & $\mathbf{t}$ & df & $\begin{array}{c}\text { Test Value }=0 \\
\text { Sig. (2-tailed) }\end{array}$ & Mean & 95\% Confidence \\
\hline & & & & Difference & Interval of the \\
\hline Post-Test Score Static Stretching & 9.665 & 9 & .000 & 32.65000 & Difference Lower \\
\hline Post-Test Dynamic Stretching & 23.599 & 9 & .000 & 31.07000 & 25.0080 \\
\hline
\end{tabular}

The table above shows, the significant value between static stretching and dynamic stretching is $p<0.005$. $t$ value for post-test score for static stretching is $\mathrm{t}(9)=9.665$, whereas for the post-test of dynamic stretching, $t(9)=23.599$.

\section{Discussion}

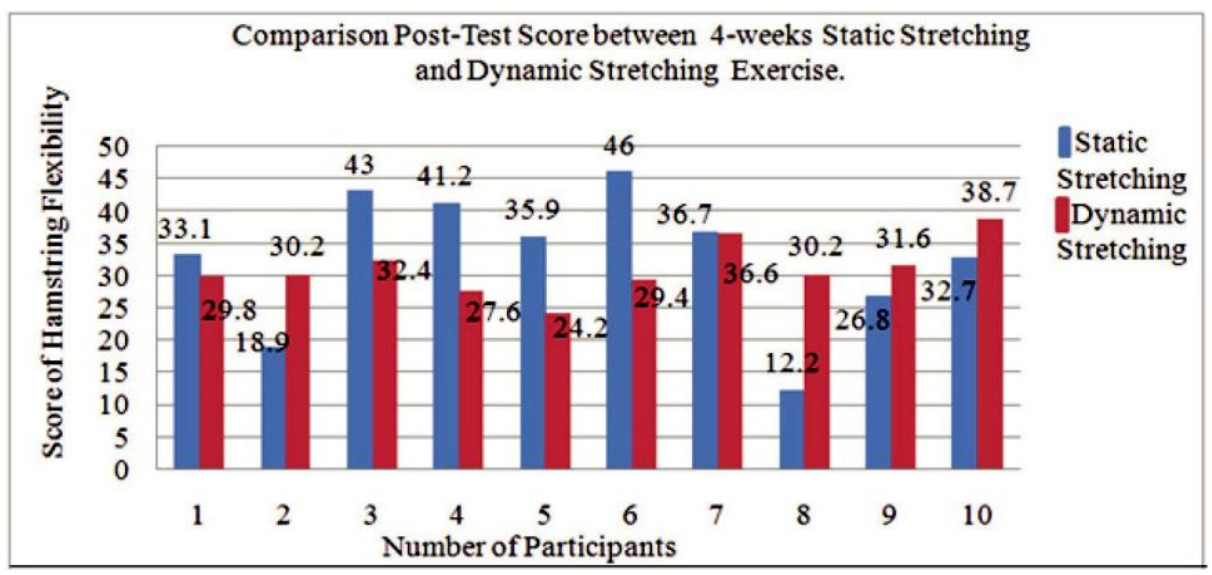

Graph 1: Comparison of the score of flexibility hamstring muscles after static stretching and dynamic stretching exercises.

Based on the diagram above, the score hamstring muscles flexibility is improved after the static stretching exercises for the first 14 people. While the scores for six more subjects show, the dynamic stretching exercises score is higher than static stretching exercises. The different between the score happens to several factors. Based on previous 
studies, exercise more static slack helps in increasing the flexibility of the hamstring muscles. This is because, static stretching exercises focusing on one particular muscle or one group muscle only J. Brent Feland et. al., 2001. No other muscles involved during stretching. Also, no additional movement that occurs during static stretching is done. Physiologically, when a muscle stretched, muscle spindle will elongate. When we focus on giving stretch to the muscles, the muscles will elongate well. It differs with dynamic stretching. Dynamic stretching requires the whole body for the movements during implementation (D. Scott Davis et. al, 2005). And this factor will increase the body temperature, increase motor unit excitability, improve kinesthetic awareness and also maximize the range of motion. Indeed, we focus on the hamstring muscles, but with whole body movement can cause muscle stretch that became the focus turns to other muscles. Technical factors also play a role. Techniques stretch the muscle while doing the stretching also needs to correct. If the method used is changing every time, the score obtained will also change each time stretching done. For dynamic stretching exercises, proper technique while doing stretching is important. If the technique used is not correct, then the other muscles will be stretched, not the target muscle groups that will elongate.

Warming up exercise is also important before doing stretching exercises. Scores flexibility will have little change if the subject refuses to warm up before doing stretching exercises. Warming up exercise had a positive effect on the muscles, joints, bones and tendons. In scientific, warming up exercises will prepare all the components of fitness before performing a specific activity. When the muscles are already warm, it's much easier to be stretched compared to muscles that are not ready. The range of motion is often associated with flexibility. Someone who has a good flexibility has a good range of motion. The range of motion is important to ensure the movement of a muscle in a good condition. Someone who has lower value of flexibility will have a limited range of motion. Limited range of motion can also cause injury. Injuries can be overcome by doing stretching exercises and workout warming up before running a specific activity. The weather and the time of execution of stretching exercises also affect the data collected. Cold weather causes the muscle is not ready. It also causes the hamstring muscle flexibility will decline at this time. The timing of stretching exercises, morning or afternoon causing scores obtained by changing the subject. In the morning, all the muscles are not ready to be stretched. Muscle spindles are not prepared with activities and caused scores obtained from the previous reduced. Different flexibility score they get when the stretching in the evening. The results are better than with the flexibility of the hamstring muscles when done in the morning. There are some subjects who suffer an injury as a result of other activities, outside of research activities. The participant scores decreased after getting injured. Despite the injuries suffered only minor injuries such as delay muscle soreness (DOMS) and muscle sprain, however, that such things can cause decreased hamstring muscle flexibility score. Injuries cause of limited movement. For the risk of an injury dynamic stretching exercises are more likely to cause injury. The additional motion caused many muscles involved. The forced movement can cause injury to someone. During stretching exercises conducted, there are some subjects who complained of injuries in the leg after doing dynamic stretching exercises. Injuries that frequently occur on the subject are muscle sprain. This statement reinforces the hypothesis stated that dynamic stretching exercises are more prone to injury than static stretching exercises. Compare to static stretching, no subject complain pain after the stretching. Same as research by Faigenbaum et al. 2005 that stated the static stretching can reduce the risk of injury potential.

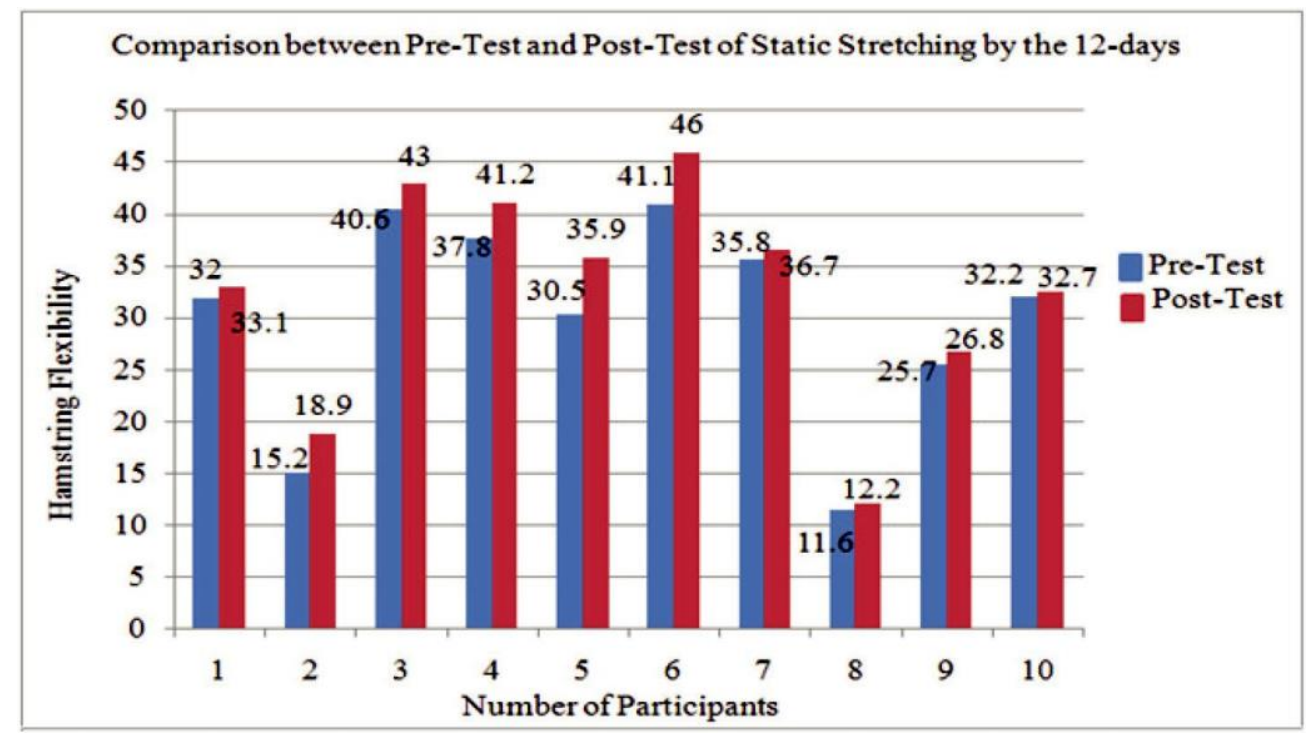

Graph 2: Comparison of hamstring muscle flexibility scores before and after the execution of 4-weeks static stretching exercises. 
The figure above shows that there is an increase in the hamstring muscle flexibility scores for each subject. A significant difference can be seen at every subject. Although there are some subjects, that have slightly increased, but the increase is still there.

\section{Comparison between Pre-Test and Post-Test of 4-weeks Dynamic Stretching}
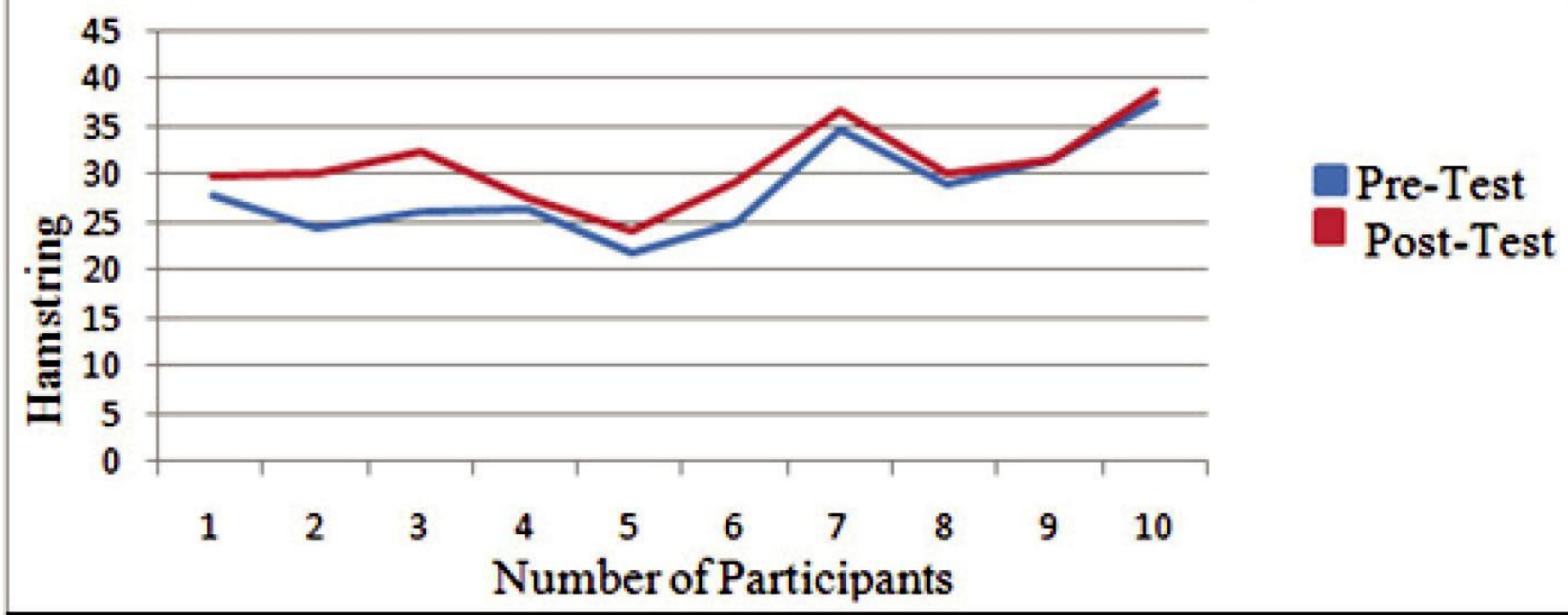

Graph 3: Comparison of hamstring muscle flexibility scores before and after performing 4-weeks dynamic stretching exercises. The figure above shows an increase of the hamstring muscle flexibility scores after doing dynamic stretching exercises for four weeks. Although there are some differences, however, its value continues to incline.

Based on the results obtained, static stretching exercises show improvement over dynamic stretching exercises. Based on previous studies also, static stretching exercises are often used as stretching exercises before doing sportspecific activities (Mohammad Taghi et. al., 2011). Election as stretching exercises also because it is easy to implement and secure. None of the subjects complained of pain or injury after perform static stretching exercises over four weeks. This statement proves that these stretching exercises are appropriate and do not cause injury. In the study that I run, score the flexibility of the hamstring muscles increased after static stretching exercises. Range of motion is often associated with flexibility. When flexibility increases, the range of motion also increase. It is because both are interrelated. In a study conducted by (Mohammad Taghi et.al., 2011) static stretching exercises have a positive impact on the range of motion. The result proves that static stretching exercises are suitable to be used to improve the flexibility and range of motion. By putting the muscle in a stretched condition and withstand the maximum possible extent the movement allowing increased muscle flexibility (William D Bandy, Jean M Irion and Michelle Briggler, 2014). Some have argued that static stretching exercises suitable performed during the cooling down process (Jose Shelton et. al., 2009). However, in my opinion, it is best done during the warming up as body workout. Their advantages in improving range of motion and flexibility and can reduce the risk of injury that might occur during sports-specific activities more are beyond dispute. Additionally, there is one study stated, this static stretching exercises indeed correspond to enhance the flexibility of the hamstring muscles. Static stretching can improve flexibility by changes in the viscoelastic properties. And it involves an increasing resultant in the length of the muscle to the viscoelastic behavior. Mohd. Waseem et.al., 2009, on his study, this type of exercise can remodel the Golgi tendon organ sensitivity position by engaging or change the series elastic component of a muscle. Changes cause elongation of the muscle components when stretching is performed on it (D. Scott Davis et. al., 2005). Based on conducted studies, conclusion can be made about dynamic stretching exercises is, these exercises can improve the flexibility of the hamstring muscles. However, when compared with static stretching, dynamic stretching exercises have the lower score than static stretching exercises. And based on the results obtained, static stretching is better to improve flexibility of the hamstring muscles than dynamic stretching exercises. Studies conducted by Brad S Based on Curry et. al., 2009, found that static stretching, dynamic stretching exercises or aerobic exercises can help improve range of motion. Dynamic stretching exercises more suitable to improve muscular performance than static stretching exercises. Dynamic stretching exercises are also often associated with the jump performance. Dynamic stretching exercises can improve performance in the high jump against static stretching exercises (Duncan and Woodfield, 2006 and Faigenbaum et. al., 2005). Dynamic Stretching exercises can also increase muscle power, and it is more suitable for activities such a jumping and high impact activities. Due to this factor, (Jose Shelton et. al., 2009; Abbas Fattahi- Bafghil et. al., 2012) says that dynamic exercise suitable exercises for warming the body. In addition, 
dynamic stretching exercises can help improve explosive performance. Many studies have found that dynamic stretching exercises that are more suitable to improve performance compared to increase flexibility and range of motion. Implementation of static stretching exercises are so easy and safe, to reduce further the risk of injury when they are implemented (Mohammad Taghi et. al, 2011). Slow movement allows and controlled movements stretching done safely without any injuries compared to other types of stretching exercises (Smith 1994). Study of Faigenbaum et al. 2005 found that static stretching can decrease the potential from getting injuries. But a study conducted by Fredrick G. A, 2000, a part of increased body temperature, increased range of motion, increase muscular performance; dynamic stretching exercises can also reduce the risk of injury. However, based on my studies, it was different because there are some subjects who suffered injuries after doing dynamic stretching exercises. So, based on my study, conclude, the dynamic stretching exercise cannot reduce the risk of getting injuries.

\section{RECOMMENDATION}

Further recommendation can be suggested to this study. There are some aspects that were not given the convergence of subjects used as sex, duration of the study conducted when the study and the type of exercise. That is because; of all the above aspects affect the results obtained. Also, improvements in terms of the number of subjects should also be considered. Time study and the number of subjects play an important role in getting better results. I also suggest that future studies can be performed using different population like children, adults or the elderly. Gender differences may also give different results on hamstring muscle flexibility. Greater emphasis should be given to the athletes so that they can know what kind of stretching exercises that are more suitable for them according to their type of game.

\section{CONCLUSION}

Conclusion, the static and dynamic stretching exercises can increase hamstring muscle flexibility. However, based on studies that have been conducted, static stretching exercises is better in improving the flexibility of the hamstring muscles than dynamic stretching exercises that focus on enhancing the quality of the performance. Last but not least, in this study, I found that static stretching exercises can reduce the risk of injury.

\section{ACKNOWLEDGEMENT}

I would like to express my sincere appreciation to my research supervisor, Mr. Srinivas Mondam for his precious guidance, passion and support in every stage of my research. This study represents a great deal of time and endeavor not only on my part but also on the part of my supervisor. He had helps me so much while completing this research until the end. Last but not least, I would like to thank to everyone who had involved in my studies as direct or indirectly which help me much and deliver for me assistance in completing this studies successfully.

\section{REFERENCES}

Bafghi, A., \& Khorasani, M. (2012). International Journal of Sports Studies. Effects of Static and Dynamic Stretching during Warmup on Vertical Jump in Soccer Players, 2 (10)(ISSN 2251-7502), 484-488.

Brad, S., Devendra, C., Gordon, J., Michelle, R., \& Patricia, J. (2009). Acute Effects Of Dynamic Stretching, Static Stretching, And Light Aerobic Activity On Muscular Performance In Women.Journal of Strength and Conditioning Research, 23(6), 18111819.

D. Scott, D., Paul, E., Kristi, L., Jerry, A., \& Jaime, M. (2005). The Effectiveness of 3 Stretching Techniques On Hamstring Flexibility Using Consistent Stretching Parameters. Journal of Strength and Conditioning Research, 19(1), 27-32-27-32.

David, G., \& Anis, C. (2011). A review of the acute effects of static and dynamic stretching on performance. European Journal of Applied Physiology, 111(11)(1439-6319), 2633-2651-2633-2651.

Jose, S., \& G. V. Praveen, K. (2009). Comparison between static and dynamic warm-up exercise regimes on lower limb muscle power. Journal of Health, Vol.1, No 2, 117-120.

Mohammadtaghi Amiri, K., Noor A., A., \& Ashril, Y. (2011). Acute Effect of Static and Dynamic Stretching On Hip Dynamic Range Of Motion During Instep Kicking in Professional Soccer Players. Journal of Strength and Conditioning Research, 25(6), $1647-1652$.

Stephen, B., Julie, G., Donna, F., \& C. Dexter, K. (2004). The Impact of Stretching on Sports Injury Risk: A Systematic Review of the Literature. Of the American College of Sports Medicine, 04(0195-9131), 3603-0371.

Types of Stretching.(n.d.). Retrieved December 20, 2014, from http://web.mit.edu/tkd/stretch/stretching_4.html

Types of Stretching.(n.d.). Retrieved December 20, 2014, from http://web.mit.edu/tkd/stretch/stretching_4.html\#SE C30

Waseem, M., Shibili, N., Ram, C., \& Faheem, A. (2009). A Comparative Study: Static Stretching Versus Eccentric Training on Popliteal Angle in Normal Healthy Indian Collegiate Males. International Journal of Sports Science and Engineering, Vol. 
ISSN 1750-9823), Pp. 180-186.

William, D., Jean, M., \& Michelle, B. (2014). The Effect of Time and Frequency of Static Stretching on Flexibility of the Hamstring Muscles. Journal of Physical Therapy Association, 77, 1090-1096.

-- 0 -- 